la discapacidad visual consecuente en la toxocariasis ocular, la cual debe siempre considerarse como diagnóstico diferencial con tumores de la retina como el retinoblastoma ${ }^{(2)}$.

Como hemos sugerido previamente ${ }^{(2)}$, sería de gran importancia que las autoridades de salud nacionales consideraran la relevancia epidemiológica de su registro en términos de morbilidad y mortalidad, ausentes en los reportes epidemiológicos correspondientes, con el fin de mejorar la comprensión de la epidemiología de la enfermedad en América Latina y avanzar así en el diseño de políticas en salud pública. Conocer mejor la enfermedad, incluyendo el mejorar su diagnóstico, permite una mejor prevención y control de esta importante enfermedad infecciosa en nuestra región.

\section{Conflictos de Interés}

Los autores declaran no tener conflictos de interés en la publicación de este artículo.

\section{REFERENCIAS BIBLIOGRÁFICAS}

1. Roldan W, Espinoza YA, Huapaya PE, Jimenez S. Diagnóstico de toxocarosis humana. Rev Peru Med Exp Salud Publica. 2010; 27(4):613-620.

2. Delgado 0 , Rodriguez-Morales AJ. Aspectos clínicoepidemiológicos de la toxocariasis: una enfermedad desatendida en Venezuela y América Latina. Bol Mal Salud Amb. 2009;49(1):1-33.

3. Dziemian E, Zarnowska H, Kolodziej-Sobocinska M, Machnicka B. Determination of the relative avidity of the specific IgG antibodies in human Toxocariasis. Parasite Immunol. 2008;30(3):187-190.

4. Coraspe V, Baptista R, Guerra I, Rivas M, Silva S, Fernandez $J$, et al. Detección de casos activos de Toxocariosis visceral mediante la prueba de Elisa-AvidezIgG. Parasitol Latinoam 2005; 60: T248-T249. (Resumen de Congreso).

5. Delgado O, Ortegoza J, Coraspe V, Rodriguez-Morales AJ. Toxocariasis phase-specific diagnosis in children from Venezuela rural areas using specific IgG antibodies relative avidity. Int J Antimicrob Agents. 2009; 34(Supplement 2):S64.

Correspondencia: Alfonso J. Rodríguez-Morales.

Dirección: Sección de Inmunoparasitología, Piso 1, Instituto de Medicina Tropical, UCV, Ciudad Universitaria. Caracas 1050, Venezuela. E.

Correo electrónico: alfonsorm@ula.ve

\section{¿TRANSMISIÓN VERTICAL O CIRCUNSTANCIAL? \\ [VERTICAL OR CIRCUMSTANTIAL TRANSMISSION?]}

José Luis Sandoval Gutiérrez ${ }^{1, a}$

Sr. Editor. Vásquez Rubén D et al. publican el primer caso de probable transmisión vertical del virus de Influenza A $(\mathrm{H} 1 \mathrm{~N} 1)^{(1)}$. Aunque teóricamente es posible la transmisión del virus por esa ruta, haciendo un paralelo con lo documentado para influenza A H5N1, actualmente no hay evidencia científica de este contagio en la literatura mundial para H1N1 como bien señalan los autores.

Si bien es una gran limitante el no haber realizado los exámenes complementarios mencionados (aislamiento viral, los hallazgos histopatológicos en la placenta), hubiese sido de gran valor el aislamiento del virus en aspirado traqueo-bronquial, ya que este es más específico ${ }^{(2)}$. El hallazgo de un PCR positivo en un hisopado nasal dentro del contexto de una pandemia no necesariamente indica que esta sea causa de la infección de la vía aérea inferior.

Es de celebrar que tanto la madre como el niño salieron avante de esta comprometida situación, felicitamos a sus médicos por su compromiso con la ciencia y la atención clínica mostrada.

\section{Conflictos de Interés}

Los autores declaran no tener conflictos de interés.

\section{REFERENCIAS BIBLIOGRÁFICAS}

1. Vázquez RD, Chávez VM, Gamio IE, Muñoz RI, Polar MF, Montalvo R, Ticona E. Probable Transmisión Vertical del Virus de la Influenza A (H1N1): A Propósito de un Caso. Rev Peru Med Exp Salud Publica. 2010;27(3): 466-69.

2. Yuelong Shu, Hongjie Yu, Dexin Li. Lethal Avian Influenza A (H5N1) Infection in a Pregnant Woman in Anhui Province, China. N Engl J Med. 2006; 354 (913):1421-1422.

Correspondencia: José Luis Sandoval Gutiérrez. Dirección:Tlalpan 4502, Col. Sec XVI, Del.Tlalpan México D.F. México. CP 14080.

Teléfono: 548-71700; Ext. 5119.

Correo electrónico: sandovalgutierrez@gmail.com

\footnotetext{
1 Departamento de Medicina Crítica, Instituto Nacional de Enfermedades Respiratorias.

a Neumólogo Intensivista. Recibido: 03-01-11 Aprobado: 12-01-11
} 